\title{
Investigating Miniature Electrodynamic Tethers and Interaction with the Low Earth Orbit Plasma
}

\author{
Iverson C. Bell ${ }^{1}$, Kyle A. Hagen ${ }^{2}$, Vritika Singh ${ }^{3}$, Steven L. McCarty ${ }^{4}$, James W. Cutler ${ }^{5}$, and Brian E. Gilchrist ${ }^{6}$ \\ The University of Michigan, Ann Arbor, MI 48109 \\ Jesse K. McTernan ${ }^{7}$ and Sven G. Bilen ${ }^{8}$ \\ The Pennsylvania State University, University Park, PA 16802
}

\begin{abstract}
The sub-kilogram, "smartphone"-sized satellite is a transformative concept, inspired by the success of nanospacecraft $(1-10 \mathrm{~kg})$ and millimeter-scale wireless sensor network concepts. These ultra-small satellites, known as picosatellites (100 g-1 kg) and femtosatellites $(<100 \mathrm{~g})$, show potential to be less costly to manufacture and boost into orbit. Thus, it may be possible to launch them in large numbers, enabling unique capabilities. Organized "fleets" of pico- or femtosatellites, however, will need a high level of coordination and maneuverability capability (i.e., propulsion). Also, many of these satellites can have a high area-to-mass ratio, which results in a short orbital lifetime in low Earth orbit due to atmospheric drag. In this paper, we summarize studies that found that short (few meters), semi-rigid electrodynamic tethers can provide 10-g to 1-kg satellites with complete drag cancellation and the ability to change orbit. We also present progress on the Miniature Tether Electrodynamics Experiment (MiTEE), currently in development. The goal of MiTEE will be to demonstrate miniature electrodynamic tether capabilities in space and study the fundamental dynamics and electrodynamics of the propulsion system.
\end{abstract}

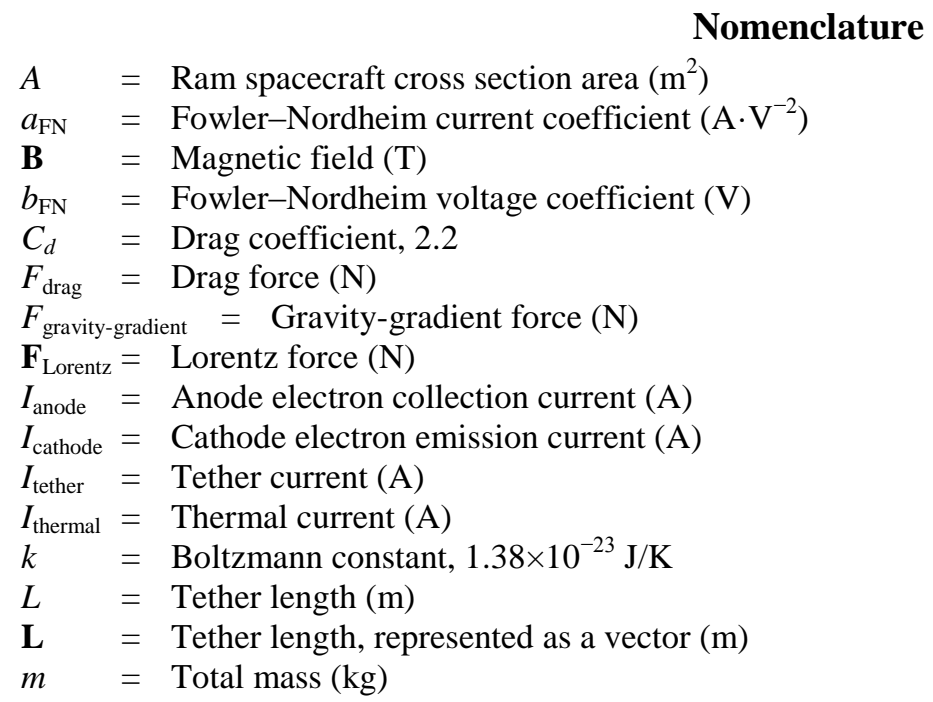

${ }^{1}$ Ph.D. Candidate, Electrical Engineering, 1301 Beal Ave., AIAA Student Member, icbell@umich.edu.

${ }^{2}$ Graduate Student, Space Engineering, 2455 Hayward St.

${ }^{3}$ Graduate Student, Space Engineering, 2455 Hayward St.

${ }^{4}$ Graduate Student, Space Engineering, 2455 Hayward St.

${ }^{5}$ Assistant Professor, Aerospace Engineering, 1320 Beal Ave., AIAA Associate Fellow.

${ }^{6}$ Professor, Electrical Engineering and Space Science, 1301 Beal Ave., AIAA Associate Fellow.

${ }^{7}$ Ph.D. Candidate, Aerospace Engineering, 229 Hammond Bldg., AIAA Student Member.

8 Assoc. Professor, Engineering Design, Electrical Engineering, and Aerospace Engineering, 213 Hammond Bldg., AIAA Associate Fellow. 
$R_{0} \quad=$ Distance from spacecraft center of mass to the Earth's center (m)

$q=$ Elementary charge, $1.6 \times 10^{-19} \mathrm{C}$

$T_{e} \quad=$ Electron temperature $(\mathrm{eV})$

$V_{\text {gate }}=$ Cathode base-gate voltage $(\mathrm{V})$

$V_{\mathrm{emf}}=$ Electromotive force $(\mathrm{V})$

$v \quad=$ Velocity $(\mathrm{m} / \mathrm{s})$

$\mu \quad=$ Standard gravitational parameter of Earth, $3.986 \times 10^{14} \mathrm{~m}^{3} \cdot \mathrm{s}^{-2}$

$\rho \quad=$ Atmospheric neutral density $\left(\mathrm{kg} \cdot \mathrm{m}^{-3}\right)$

$\Phi_{p} \quad=$ Probe potential $(\mathrm{V})$

\section{Introduction}

$\mathrm{T}$ he sub-kilogram, "smartphone"-sized satellite is a transformative emerging concept inspired by the success of nanospacecraft $(1-10 \mathrm{~kg})$ and millimeter-scale wireless sensor network concepts (i.e., "SmartDust"). ${ }^{1-6}$ The continuing interest in very small spacecraft with longest dimension in the tens of centimeters and now even smaller, down to a few centimeters, is motivated by growing capabilities to integrate more functionality and sophistication into an ever smaller volume. Much of this early enhancement in capability has come from electronics miniaturization and power reduction. Improvements in integrated circuit and microelectromechanical systems (MEMS) technology have made possible satellites at the levels of fully monolithic semiconductor integrated circuits $(10-100 \mathrm{mg})$ or hybrid integrated circuits (10-300 g). Effectively, this architecture can be thought of as a small "satellite-on-a-chip," or ChipSat. These satellites, categorized as picosatellites (100 g-1 kg), femtosatellites $(<100 \mathrm{~g})$, and here, more broadly, as "ultra-small satellites" ( $\leq 1 \mathrm{~kg})$, represent the next frontier in miniaturization.

This trend suggests that matching or exceeding the capabilities of today's nanosat could be achievable near and below the 100-gram level (femtosats). Pico- and femtosatellites show potential to be less costly to manufacture in bulk and boost into orbit because of their low mass and small size, ${ }^{7}$ so it may be possible to launch them in large numbers, possibly enabling unique mission capabilities. Several studies describe applications of pico- and femtosatellite constellations, like global monitoring of natural disasters and investigating ionosphere scintillation. ${ }^{8-13}$

However, missions using organized "fleets" of pico- or femtosatellites would need a high level of coordination, and maneuverability (i.e., propulsion). Flat ChipSats also have an inherently high area-to-mass ratio. Many of the suggested designs take on a flat, planar shape because components are mounted on printed circuit boards (PCBs) and/or silicon wafers. Although the high area-to-mass ratio can be exploited for unique dynamics, it can result in a short lifetime in low Earth orbit (LEO) due to atmospheric drag, ranging from a few days to a few hours depending on altitude and solar conditions. ${ }^{14}$ Therefore, propulsion is needed to increase mission lifetime. Tracking, communication, energy generation, and energy storage also present challenges due to their small size.

A variety of concepts have been proposed for picoand femtosatellite propulsion. However, while a satellite using consumable propellant (e.g., a miniaturized cold gas thruster or solid propellant system) can overcome atmospheric drag, the volume/mass of required propellant will increase unacceptably as the desired satellite lifetime increases. $^{14}$

Previous trade studies have shown that a short (few meters length), semi-rigid electrodynamic tether has potential to provide propellantless propulsion for ultrasmall satellites. ${ }^{14-17}$ The concept is shown in Fig. 1. ED tethers can also be used for harvesting electrical energy from the orbit, allowing for propellantless, self-powered deorbiting. Furthermore, this same tether could serve as an enhanced communication or scientific radio antenna aperture. In this paper, we summarize studies investigating how short tethers can enhance ultra-small satellite capabilities and share developments in the Miniature Tether Electrodynamics Experiment (MiTEE)

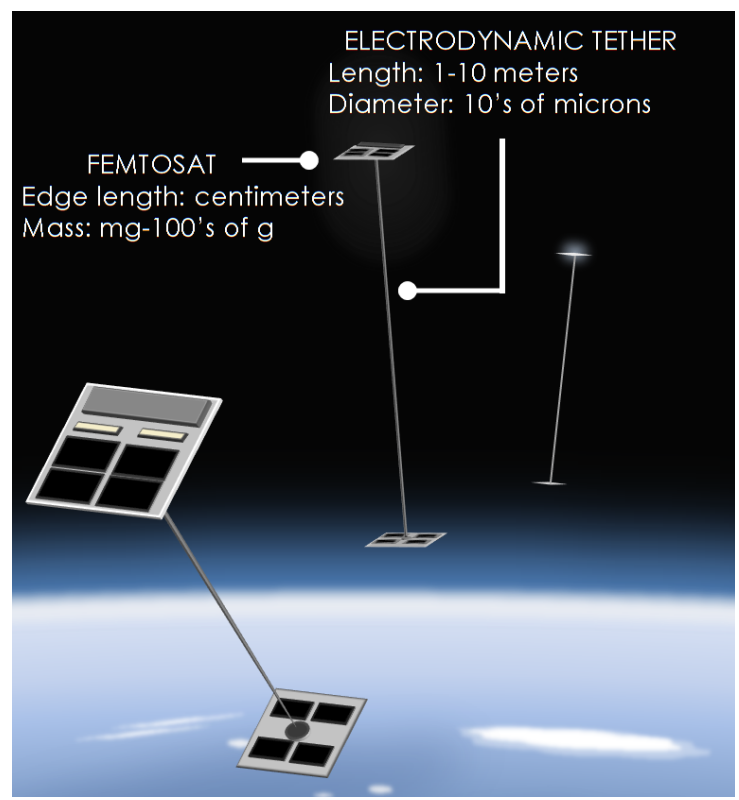

Figure 1. Concept of ED tethers with pairs of femtosats as a maneuverable, coordinated fleet. ${ }^{14}$ 


\section{Preliminary Trade Studies}

\section{A. Electrodynamic (ED) Tether Background}

Electrodynamic tethers (ED tethers) are often thought of as long conducting cables, often times 100s of meters to kilometers in length. When the cable conducts current in the presence of a planetary magnetic field, the interaction of the tether current with the planetary magnetic field produces a force that allows the tethered satellite system to boost, deboost, and change inclination without propellant and scavenge energy from the spacecraft orbit. This force is expressed as

$$
\mathbf{F}_{\text {Lorentz }}=\int_{0}^{L} I_{\text {tether }} d \mathbf{L} \times \mathbf{B} \text {. }
$$

Current conducted by the tether is collected by a satellite at one end of the tether while a satellite at the opposite end emits current. Final circuit closure occurs in the ambient plasma, satisfying Kirchhoff's Voltage Law. Figure 2 shows an illustration of the basic components in the concept.

\section{B. System Concept Description}

The miniaturized ED tether considered here is a short (several meters), insulated-but-conducting tether connecting a pair of nearly identical pico- or femtosatellites that work together as a unit. Figure 2 shows an illustration of the basic concept. Each satellite is equipped with a solar panel, power supply, cold cathode electron emitter, and is capable of collecting electrons on the surface. Although several satellite sizes have been considered in previous trade studies, this report highlights the results of the $10 \mathrm{~cm} \times 10 \mathrm{~cm} \times 1.25 \mathrm{~cm}, 100$-g satellite.

The ED tether assumed here is considered a "semi-rigid" structure. This is in contrast to much longer tether systems with massive end-bodies, the gravity-gradient tension force assists with deployment and keeps the tether nearly straight in the presence of lateral forces (e.g., drag or solar radiation pressure) along its length and on the end-bodies. Here, the gravity-gradient force will be small for ED tethers on the ultra-small satellite scale, so a material with an appropriate level of shape memory is desired for the tether to establish and hold its shape once deployed on orbit. However, the conducting strands should also be flexible enough to be collapsed for storage until deployed on orbit.

The ED tether prototype considered here has a Monel ${ }^{\mathrm{TM}}$ core to carry current and provide the needed level of rigidity. A thin layer of Teflon ${ }^{\mathrm{TM}}$ provides insulation. The tether's radius increases with length to provide a higher area moment of inertia and thus rigidity at longer lengths. ${ }^{15}$

\section{Electron Collection and Emission}

Field emitter array cathode (FEAC) technology can be used to emit electrons at one end of the tether. Conventional Spindt-tip FEACs were developed by SRI International in the late 1960s and feature sharp molybdenum cones on the scale of one micron in diameter as emitter tips ${ }^{18} \mathrm{~A}$ biased grid is used to establish an electric field (on the order of single $\mathrm{V} \cdot \mathrm{nm}^{-1}$ ) at the emitter tips that facilitates electron emission. Arrays of vertically aligned carbon nanotubes can also be used as an alternative to molybdenum cones. ${ }^{19}$ Carbon nanotube cathodes may provide lower power consumption requirements, with electric field magnitudes required to emit electrons ranging from $1-10 \mathrm{~V} \cdot \mu \mathrm{m}^{-1}$ as opposed to around $100 \mathrm{~V} \cdot \mu \mathrm{m}^{-1}$ for Spindt-tip cathodes. ${ }^{20}$ Robustness in the space environment remains an important question to answer.

FEACs obey the Fowler-Nordheim emission law for current emission, 


$$
I_{\text {cathode }}=a_{\mathrm{FN}} V_{\text {gate }}^{2} \exp \left(-b_{\mathrm{FN}} / V_{\text {gate }}\right) .
$$

For a given emission area, there is a maximum space-charge-limited current density that is possible based on emission energy and ambient plasma conditions, although the current densities considered in this trade study are below that limit. ${ }^{14}$

On the opposite end of the tether, it is assumed that the outer surface of the pico- or femtosat can be coated in a transparent conductor, such as indium tin oxide (ITO), and biased above the plasma potential to collect electrons. The possible shape of the pico- and femtosatellite, the relative motion of the plasma, and the presence of an ambient magnetic field add complexity to predicting collection current. However, simplifying assumptions, described in Ref. 13, were made to estimate the current. The expression for anode current, ${ }^{21}$

$$
I_{\text {anode }}=\frac{I_{\text {thermal }}}{2}\left(1+\frac{q\left(V_{\text {anode }}-\Phi_{p}\right)}{k T_{e}}\right)^{\beta},
$$

was developed empirically from measurements in LEO. The dimensionless parameter $\beta=0.85$ was chosen to be conservative.

\section{Experimentally Refining Electron Collection Estimates}

The plasma contactors are critical components of ED tether propulsion because they close the circuit in the ambient plasma, allowing current to be conducted in the tether. Thus, it is important for the plasma contactors to have a well characterized current-voltage relationship. The efficiency of the tether-plasma interface for current collection and emission is often the limiting factor in the overall ED tether system efficiency.

Estimating the electron collection current to the surfaces of a pico- or femtosat is complex. In previous studies, simplifying assumptions were made to estimate current. We are working to refine our anode current collection estimate by conducting ground-based plasma experiments that capture critical characteristics of the LEO environment. The ground-based experimental facility will be the cathode test facility (CTF) at University of Michigan's Plasmadynamics and Electric Propulsion Laboratory. A flowing plasma will be generated by a lanthanum hexaboride $\left(\mathrm{LaB}_{6}\right)$ hollow cathode, which simulates the relative velocity between the ionospheric plasma and the orbiting satellite. The goal is for key plasma parameters, such as the Debye length to probe size, to scale with ionospheric plasmas. ${ }^{22}$

\section{E. Power Generation Estmates}

The power generated on-board the pico- or femtosat is limited, so it is important to determine if a pico- or femtosat can generate the power necessary for drag make-up or thrust. The power required by the anode and cathode are a majority of the electrical demand. Figure 3 compares the estimated power demand to the estimated power generated for propulsion. Following the approach outlined in Ref. 23 , it was estimated that roughly $3.4 \mathrm{~mW} \cdot \mathrm{cm}^{-2}$ are generated for propulsion.

The overall onboard power generation estimate is conservative, following an assumption that the solar cells have an energy conversion efficiency of $10 \%$. We also assume that $80 \%$ of the generated power can be used for propulsion and $15 \%$ of this power is dissipated in step-up voltage DC-DC converters and other loads. For the 100-g satellite, we assume that both large, flat areas of each satellite are equipped with solar cells, one side facing the Sun out of eclipse while the Earth-facing side collects Earth albedo. The power generated from only one of the satellites is used

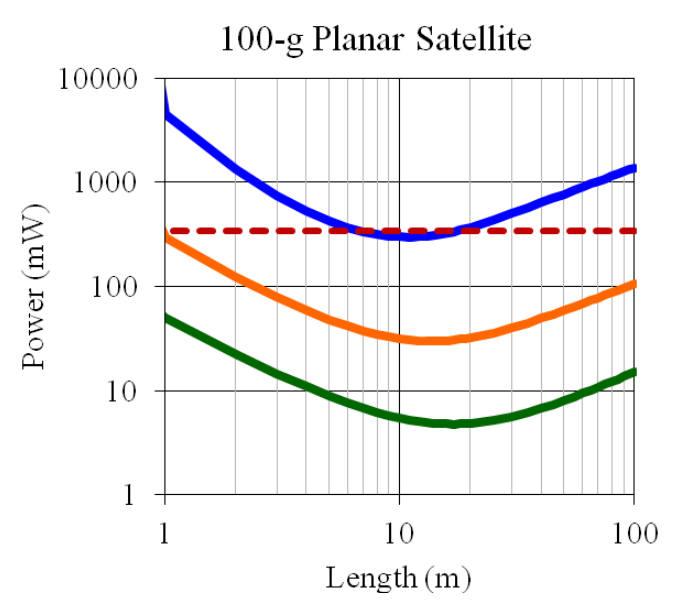

Figure 3. Estimated power needed for drag makeup at $400 \mathrm{~km}$ (green), $500 \mathrm{~km}$ (orange), and $600 \mathrm{~km}$ (blue) and power available for propulsion (red). ${ }^{17}$ 
to generate thrust (see Fig. 2). The result is that the 100-g satellite is estimated to have about $345 \mathrm{~mW}$ of power available for propulsion. ${ }^{17}$

At each altitude, there is a tether length that minimizes the required drag make-up power. Although extremely short ED tether lengths may be easier to store and deploy, they require a large current to overcome drag. High current translates into relatively high power dissipated in the tether, anode, and cathode. For a very long tether, the rigidity decreases with length, so it must have a relatively large radius to prevent bending or bowing, or be "semirigid." As a result, the drag due to the tether dominates over the drag due to the satellite, driving up the required current. The current is minimized when these two effects are balanced. This motivates us to choose a $10-\mathrm{m}$ long tether for the $100 \mathrm{~g}$ satellite. The tether current needed for drag make-up ranges from $0.1-5 \mathrm{~mA}$, varying with altitude. ${ }^{17}$

\section{F. Force Estimates}

The drag force and the gravity gradient force are the dominant perturbation forces that impact the dynamics of pico- and femtosats. The expression for atmospheric drag is given by

$$
F_{\text {drag }}=\frac{1}{2} \rho C_{d} A v^{2} .
$$

The drag coefficient $C_{d}$ is assumed to be $2.2 .^{23}$ Figure 4 reveals that the 100 -g satellite is able provide thrust equivalent or exceeding drag at $400 \mathrm{~km}, 500 \mathrm{~km}$, and $600 \mathrm{~km}$. The 10 -meter-length "sweet spot" mentioned earlier also appears to correspond to the point in which tether thrust exceeds drag by the largest margin.

The approximate magnitude of the gravity gradient force is given by ${ }^{23}$

$$
F_{\text {gravity-gradient }} \approx \frac{3 m \mu L}{R_{0}{ }^{3}} \text {. }
$$

The gravity gradient force acts vertically to create tension in a tethered system and causes a torque that provides attitude stability. The gravity-gradient force in Fig. 4 exceeds drag for a range of tether lengths. This suggests that the gravity-gradient force will ensure a degree of stability at the tether length of interest. However, the ED tethers used for pico- or femtosat propulsion are miniaturized to the scale of the satellites, and this has the unfortunate effect of reducing the gravity gradient force with the satellite size.

It will be necessary to study the relative strength of the drag and gravity-gradient torques in order to understand the resulting tether attitude. If the center of mass and center of pressure are vertically displaced in a tether system, the aerodynamic drag torque will tend to rotate the entire system until the two are parallel to the velocity vector. However, if this causes the longest axis of the satellite to rotate away from the local vertical, the gravity-gradient torque, if strong enough, will counteract the rotation and restore the tether to the local vertical.

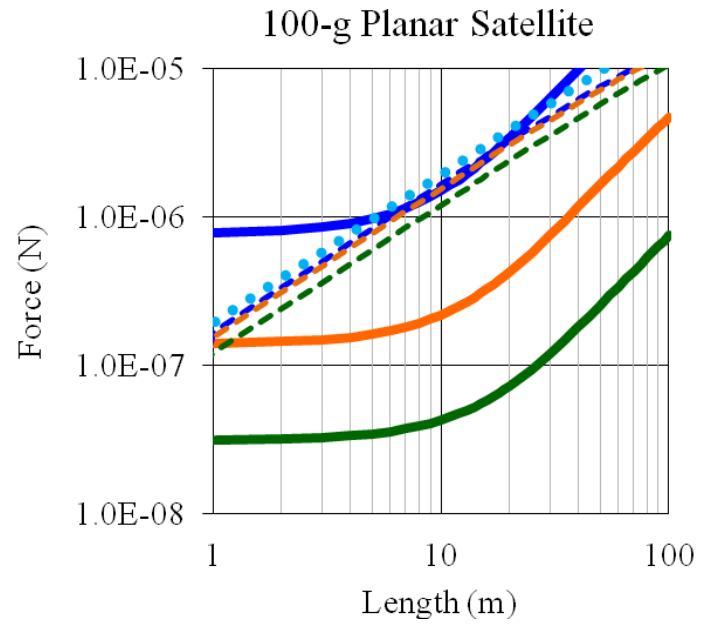

Figure 4. Estimated forces on the tethered system. The thrust forces (dashed lines) and drag forces (solid lines) at $400 \mathrm{~km}$ (blue), $500 \mathrm{~km}$ (orange), and $600 \mathrm{~km}$ (green) altitudes are shown as well as the gravity gradient force (light blue dotted line). ${ }^{17}$

\section{G. Performance Simulation}

The software tool TeMPEST allows us to simulate an ED tether system in orbit. TeMPEST incorporates geomagnetic field models, ionospheric and atmospheric conditions, plasma contactor modeling, and precise orbital calculations to predict propulsion performance. We used TeMPEST to generate Fig. 5, which shows the altitude change of the 100-g femtosat with (blue) and without (orange) the ED tether propulsion at $400-\mathrm{km}, 500-\mathrm{km}$, and 600-km starting altitudes. Rapid drag deboost can be seen without an ED tether, whereas actual boost capability is shown with a low-power, short (10 m) ED tether. 
Although the altitude curves in Fig. 5 appear to widen, this merely represents increasing eccentricity of the satellite over time. This effect is particularly pronounced for ED tethers that are continuously boosting. The thrust force increases in regions of the ionosphere where the electron density is higher, and the uneven thrust in each orbit results in an increasing orbital eccentricity. However, ED tether boosting can be planned so the satellite orbit eccentricity degradation is minimized.

\section{H. Using the Electrodynamic Tether as an Antenna}

Ultra-small satellites have inherently small antenna apertures and low transmission power, but a conducting coating (e.g., gold, copper, silver, etc.) on the semirigid tether core would provide the potential for a high performing, long, directional antenna. The conducting layer would only need to be one or a few skin depths in thickness (on the order of micrometers, depending on frequency) to radiate the electromagnetic signal with low resistive loss.

We modeled the radiation pattern of the ultra-small satellite ED tether at $430 \mathrm{MHz}$ using ANSYS $^{\circledR}$ HFSS $^{\text {TM }}$ simulation software as shown in Figure 6. The antenna can be modeled as an off-centered dipole if a short wire, 10 s of centimeters long, is attached to one of the tethered satellites. It was also found that at the CubeSat scale, the conducting satellite frame can be used in lieu of the additional short wire. The $10 \mathrm{~cm} \times 10 \mathrm{~cm} \times 10 \mathrm{~cm}$ CubeSat structure is resonant at the UHF frequency, so the off centered dipole model with quarter wavelength pole and tether connected to the CubeSat have approximately the same radiation characteristics. The $z$ axis in Fig. 6 points in the nadir direction. With a small resonator in the tether line at the proper location, the antenna can also be adjusted for frequency and gain independent of its overall length.

Thus, in practice, we can consider an ultrasmall satellite constellation with the above capabilities (A. to H.) to be something more: a carefully managed "fleet" of organized, spatially reconfigurable, capable sensor platforms. ${ }^{17}$

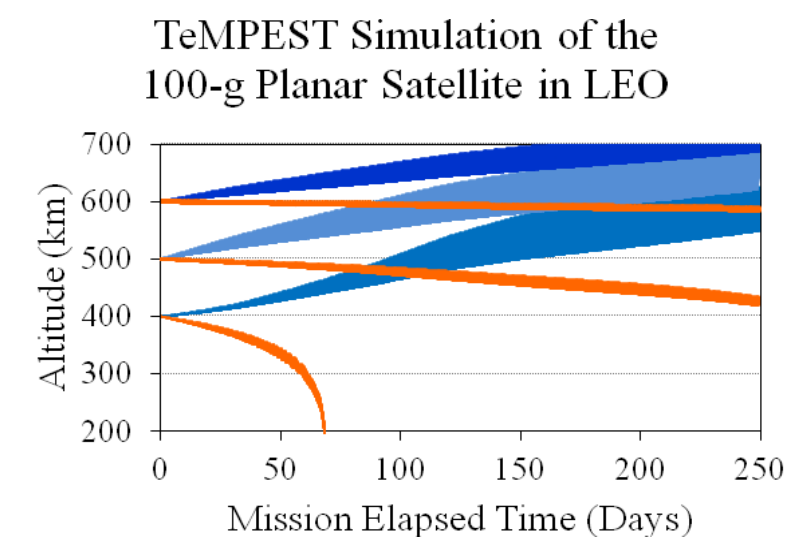

Figure 5. Simulation of a single satellite (orange) starting at $400 \mathrm{~km}, 500 \mathrm{~km}$, and $600 \mathrm{~km}$ compared with dual satellites with an ED tether (blue). ${ }^{17}$

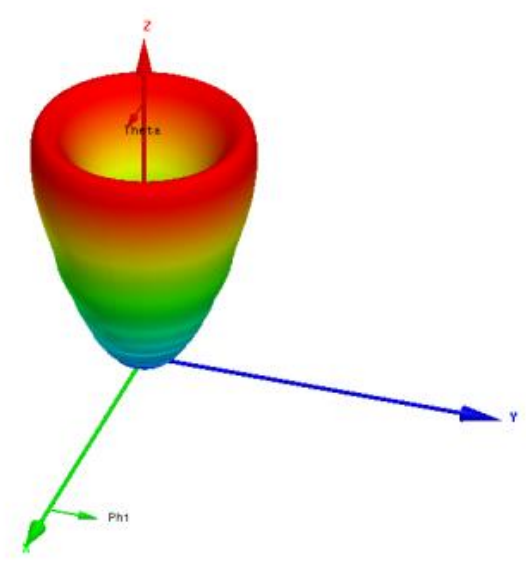

Figure 6: The 3D radiation pattern for a 10meter-long tether. ${ }^{17}$

\section{Miniature Tether Electrodynamics Experiment (MiTEE) Space Mission Description}

The Miniature Tether Electrodynamics Experiment (MiTEE) is a technology-demonstration mission that will deploy a tethered pico- or femtosat from a CubeSat and evaluate essential characteristics of a miniature ED tether. The mission will consist of two end bodies connected by a short, insulated, conducting tether, as shown in Fig. 7. While in storage, the tethered end bodies will form a $1 \mathrm{U}$ CubeSat consisting of a pico- or femtosat smaller body, with a mass of roughly $50 \mathrm{~g}$ to $250 \mathrm{~g}$ and a volume of up to $250 \mathrm{~cm}^{3}$. The larger body, with the remaining mass and volume of a $1 \mathrm{U}$ CubeSat, will house a majority of the primary subsystem equipment and will act as the deployer. The anode, biased positively to collect electrons, and the cathode, or the electron emitter, are exposed to the Earth's ionosphere to complete the "plasma circuit" and generate current within the tether. While orbiting the Earth, MiTEE will pass through the Earth's magnetic field, which when combined with the tether current, will generate a Lorentz force on the satellite system that can be used to boost or de-boost MiTEE. 
MiTEE's primary mission objectives are to assess the key dynamics of the tethered system, determine the current-voltage characteristics of the tether, and measure the presence of thrust. Furthermore, a secondary mission objective includes the use of the tether as an antenna for satellite-to-ground communication.

\section{A. Tether Electrodynamics}

Two cathode technologies, the Field Emitter Array Cathode (FEAC) and thermionic emission cathode, have been investigated for use on MiTEE. Due to the power limitations within the CubeSat platform, power consumption and operational lifetime are critical factors of the cathode design. Electron emission testing of a thoriated tungsten filament has been carried out with the material being chosen based on its ability to operate in the temperature range required to induce electron emission, while maintaining a reasonably low work function of $2.6 \mathrm{eV}$. Lower work function materials are typically preferred as filament materials because they emit electrons at lower temperatures. While $\mathrm{BaO}$ and other materials with even lower work functions were

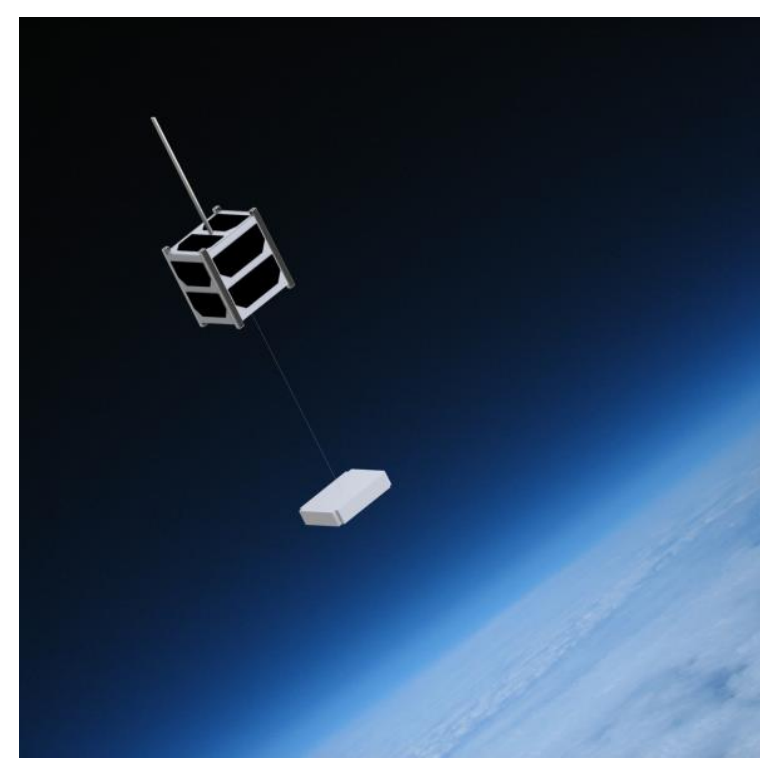

Figure 7: MiTEE concept shown with a partial deployment. ${ }^{17}$ considered, their oxidation with atomic oxygen in the Earth's ionosphere may reduce the lifetime of the cathode. Additionally, some of them require an activation process that involves heating the filament above normal operating temperature for an extended time. This process may be a large drain on the onboard electrical power.

The test apparatus used a copper mesh grid with an applied potential of $50 \mathrm{~V}$ and a filament threshold current of $300 \mathrm{~mA}$, which resulted in an emission current of approximately $5 \mathrm{~mA}$. The power consumption to achieve this current was determined to be $1.5 \mathrm{~W}$, which is within the average power collection of solar cells on MiTEE produced from simulations in AGI's System Tool Kit ${ }^{\mathrm{TM}}$ (STK). Optimization of the filament, grid, and plate specifications was not taken into consideration within the preliminary tests. Testing for power consumption is also underway on a FEAC. The cathode and anode will also require a small high voltage power supply (HVPS) capable of producing elevated voltages (50-200 V) at high efficiency.

\section{B. Orbit and Dynamics Simulations}

One of the primary mission objectives is to measure the presence of thrust on the tethered satellite system. In order to estimate the thrust of a short, 10-meter tether, simulations with various orbital parameters including altitude and inclination were run with TeMPEST. TeMPEST-calculated thrust values are provided in Fig. 8 and the associated changes in altitude are shown in Fig. 9.

Inclinations from $40^{\circ}$ to $98^{\circ}$ and altitudes from $400 \mathrm{~km}$ to $600 \mathrm{~km}$ were analyzed based on likely CubeSat launches as well as their lifetime constraints. Thrust contributing to boosting or de-boosting maneuvers, known as in-track thrust because it is within the plane of the velocity vector, increases at lower altitude and lower inclination orbits. Higher in-plane thrust will increase the ability to detect the presence of thrust on the tethered satellite system as it results in larger altitude deviations from a non-thrusting case. The average in-plane thrust was then used in STK to calculate seven-day average altitude differences between thrusting and non-thrusting cases. Short term (sevenday) altitude averages were calculated so that the timescale of force detection could be observed. At higher altitudes and inclinations, force detection will either require more accurate measurements or altitude averages over longer periods. MiTEE will be designed for use within a wide range of orbits to ensure success in detecting thrust. 


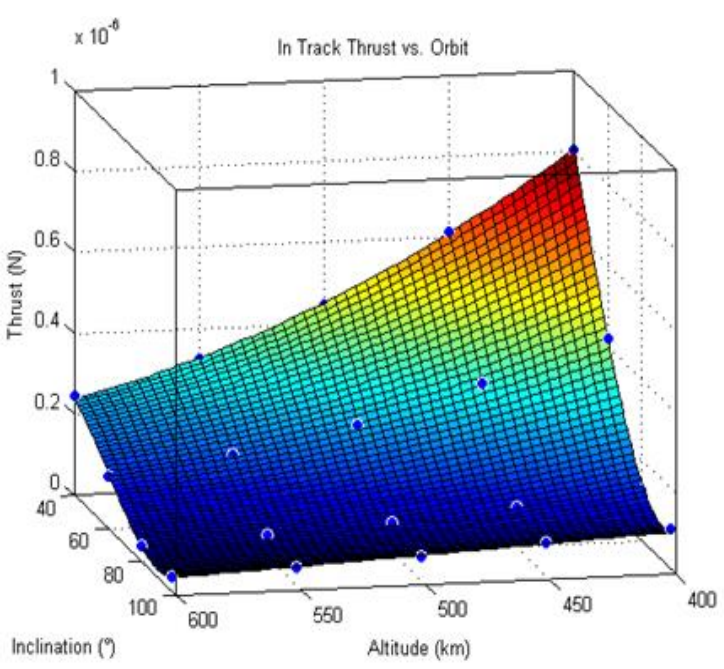

Figure 8: In-track thrust increases as altitude and inclination decreases.

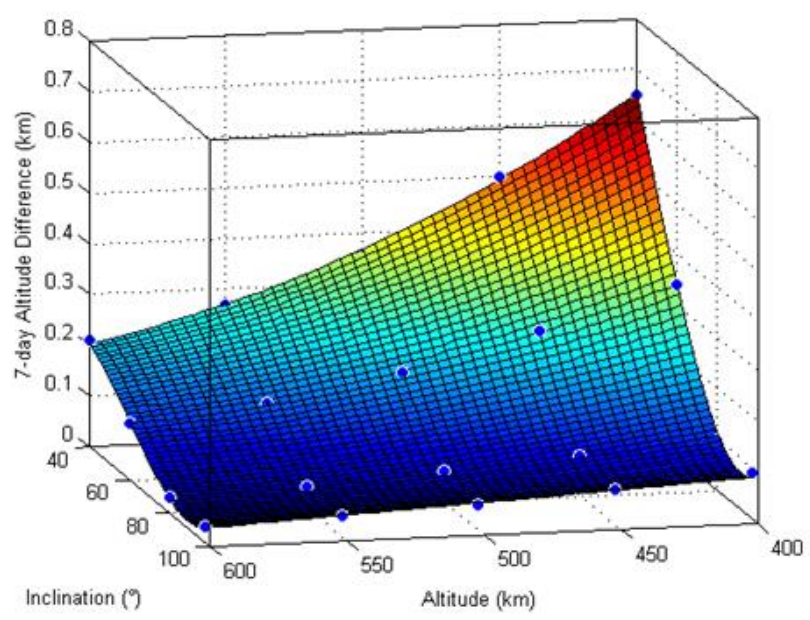

Figure 9: Seven-day altitude difference between thrusting and non-thrusting cases.

Another primary objective of the mission is to characterize the attitude dynamics of a miniature tethered satellite system. Gravity-gradient torques, drag, and the Lorentz force contribute to a motion around its local vertical. The angles of motion relative to the local vertical are called the libration angles. Preliminary simulations have been completed using TetherSim ${ }^{\mathrm{TM}}$, a tether multiphysics simulator from Tethers Unlimited, Inc., to analytically show how the tethered system will behave in orbit. A Propulsive Eletrodynamic Tether (PET) with a 10-meter flexible tether was simulated for 12 hours. A $400-\mathrm{km}$ altitude and $51.6^{\circ}$ inclination orbit were assumed. This orbit has a lower inclination, relatively good communication access to Ann Arbor, MI, and is close to the International Space Station's (ISS) orbit, which has launched CubeSats. In-plane and out-of-plane libration angles are shown in Fig. 10 without tether current flow. The configuration appears to be nearly aligned to the local vertical. The deployed body lags consistently behind the upper body, with a mean in-plane angle of approximately $0.7^{\circ}$, as measured from the local vertical. Around this mean angle, the deployed body oscillates in plane with a period of approximately 24 minutes and a max amplitude of $0.9^{\circ}$. Out-of-plane librations are much smaller, with a period of 47 minutes and an amplitude of $0.2^{\circ}$. As electrodynamics modeling was disabled for the simulation, these results do not account for perturbations due to either induced or applied currents that would generate a force that may increase the amplitude of the oscillations.

In order to meet the primary objective of studying the dynamics of the tethered system, a dynamics sensor capable of determining these libration angles and the distance between the end bodies will be required. Camera sensors have been investigated with the added benefit of having visual references of tether operation. By placing markers on the endbody, a distance measurement can be made between two or more markers due to the attitude motions of the endbody. Using a 5-megapixel camera located $2 \mathrm{~m}$ from the endbody with a $10-\mathrm{cm}$ distance between markers yielded 139 pixels between markers. At $10 \mathrm{~m}$ from the endbody, the distance between markers yielded only 22 pixels, which results in a \pm 0.33 -m resolution. An angle relative to the camera can be calculated either by its placement in the array of pixels or by lens mapping. A fisheye lens uses

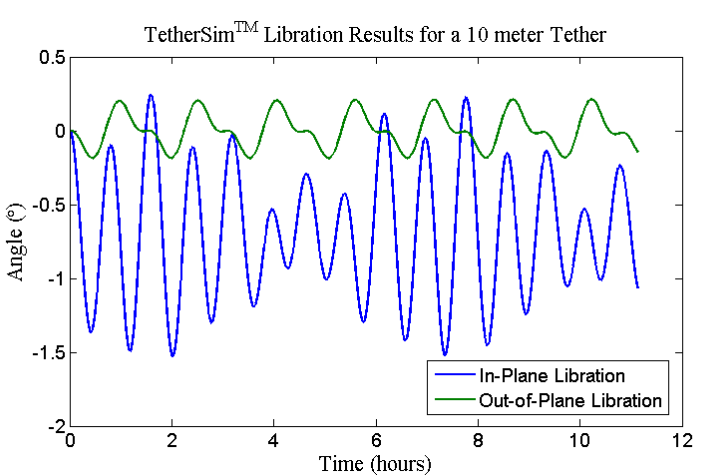

Figure 10: TetherSim ${ }^{\mathrm{TM}}$ results showing the in- and out-of-plane libration angles over time for the MiTEE tethered spacecraft. equiangular mapping to find the relative angle from the camera to an object. The fisheye lens would also have the added effect of having nearly hemispherical vision, which would allow measurement of large libration angles. 


\section{Primary Communications Analysis}

The capabilities of the primary communication system on board MiTEE were investigated because the communication system directly impacts the amount of mission-critical science data generated and transmitted. The preferred choice for frequency and antenna were amateur UHF and quarter wavelength monopole due to their extensive flight heritage. ${ }^{24}$ Simulations were carried out to study the radiation performance. The impact on the radiation pattern due to antenna placement was also studied. HFSS simulations were run for antennas mounted in different positions on each face of a $1 \mathrm{U}$ CubeSat. It was also decided to investigate if using two monopoles orthogonal to each other would give better omnidirectionality. Further tests with dual monopole antennas with orthogonal axes are planned.

Link margin simulations were run assuming ISS orbit by taking the gain pattern with a $1 \mathrm{U}$ ground plane found via HFSS simulation and then importing the pattern into STK. In order to receive data with a Bit Error Rate (BER) of $1 \times 10^{-5}$ for an FSK/GMSK modulation scheme, which is common to amateur radio communication, the ratio of energy per bit to noise power spectral density, or $\mathrm{E}_{\mathrm{b}} / \mathrm{N}_{0}$, of $10 \mathrm{~dB}$ or more is required. A margin of $3 \mathrm{~dB}$ over and above $10 \mathrm{~dB}$ is assumed as a requirement for link closure. The link margin analysis results are shown in Fig. 11. In Fig. 11, the $x$-axis points in the direction of satellite orbital motion, the $z$-axis points in the anti-nadir direction, and the $y$-axis points in the direction orthogonal to both $x$ and $z$-axes.

Several different antenna configurations were used for the link analysis: single monopole and a dual monopole (placed orthogonal to each other) fed with a $0^{\circ}, 90^{\circ}$, and $180^{\circ}$ phase shift

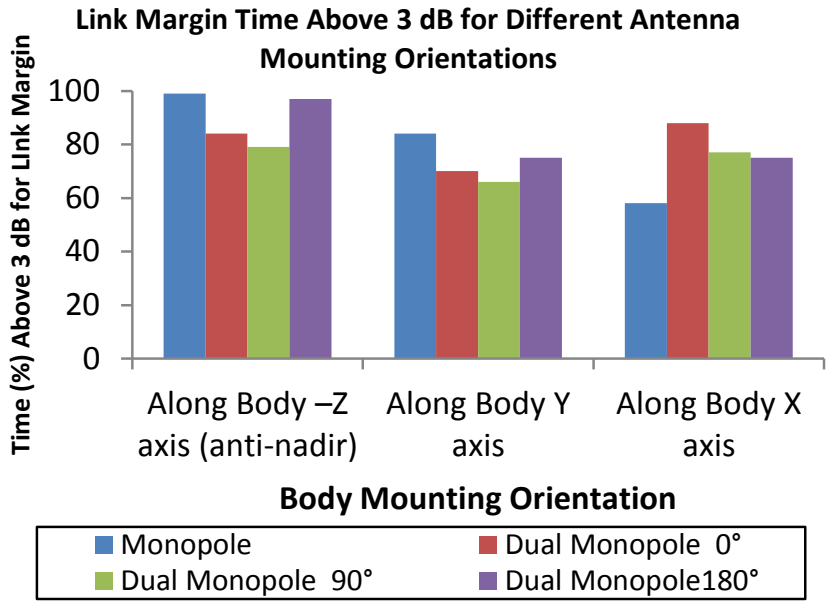

Figure 11: Primary antenna link margin times above $3 \mathrm{~dB}$ for total access time between MiTEE and the ground station.

along with different orientations around the axes of a $1 \mathrm{U}$ CubeSat. The tethered system is also assumed to be a stable along the local vertical and nadir pointing; however, a 1-dB loss associated with the tether motion is accounted for within the link budget. Even though dual monopoles have less deep nulls than the monopole antenna, the link margin time above $3 \mathrm{~dB}$ was not found to be better for all antenna mounting orientations. The simulations were run with a random rotation rate of $3 \% \mathrm{sec}$ along all axes to mimic spacecraft tumbling; however, the link margin time above $3 \mathrm{~dB}$ was roughly the same for each antenna. The monopole antenna was found to provide the highest access time above a $3-\mathrm{dB}$ margin over the dual monopoles with phase shifts.

\section{The MiTEE Spacecraft}

Since the CubeSat form factor allows for a limited amount of power collection and consumption, spacecraft components will need to be duty-cycled to conserve the amount of available power. The spacecraft will feature solar panels for power collection. Tether operation and critical mission equipment will be maintained through duty cycling to ensure that the mission is power positive. Communication to the satellite will be accomplished through a primary antenna and radio in addition to investigating the tether as a potential means of communication. Detumbling will be achieved through the use of an Attitude Determination and Control System (ADCS) capable of reducing the initial motions resulting from orbital insertion. The deployer of the pico- or femtosat body is another component critical to the mission. This deployment mechanism must safely deploy the tether without snagging or damaging it on any supporting structure. Current designs being investigated and tested include frangibolts, pinpullers, a nichrome burn system, as well as additional commercially available mechanisms. The endbody must also have limited tip-off rates to ensure that the tether does not become tangled or wrapped around the endbody.

\section{E. The Next Phase of Mission Development}

The next phase of MiTEE's development, which will continue into 2014, includes testing and further investigations of high risk and critical components associated with the mission. Further testing and modeling of the thermionic emission cathodes and FEACs will be completed to determine the operating characteristics of the cathode system. Microgravity testing of a deployment mechanism would enable the study of behaviors closer to 
those experienced in orbit than in ground testing. Modeling of the tether dynamics on deployment and power collection in orbit will also continue in the next phase of MiTEE as associated risks are reduced.

\section{F. Mission Development Student Team}

Over the course of a year, a combined 31 doctoral, masters, and undergraduate students have been involved at some point with the MiTEE project. Starting in September 2012, the team has been strengthened from the inclusion of a wide range of students with backgrounds that include aerospace, electrical, mechanical, and systems engineering. The team is led by students with advice and guidance from faculty. The concept and design of MiTEE will be further refined as the mission develops.

\section{Summary}

Studies have shown that the miniature tether concept has potential to enhance capabilities for picosatellites and femtosatellites. We believe the MiTEE mission provides an opportunity to demonstrate these capabilities in the space environment. Our vision is that the enhanced maneuverability will allow a constellation of pico- or femtosatellites to function as a coordinated fleet rather than a swarm.

\section{Acknowledgments}

The authors gratefully acknowledge support from AFOSR grant FA9550-09-1-0646, the National Science Foundation Graduate Student Research Fellowship under Grant No. DGE 1256260, the Michigan Space Grant Consortium, and the Multidisciplinary Design Program at the University of Michigan. I.C. Bell would also like to thank A. Venkitasubramony, D. Dimas, A. Cauligi, N. Sawicki, C. Lu, and K. Van Gorkom for their valuable contributions.

\section{References}

1. Kahn, J. M., Katz, R. H., and Pister, K. S. J., "Next Century Challenges: Mobile Networking for Smart Dust," Proc. ACM MobiCom '99, Washington, DC, 1999, pp. 271-78.

2. Warneke, B.A., et al., "An Autonomous $16 \mathrm{~mm} 3$ Solar-powered Node for Distributed Wireless Sensor Networks," IEEE Sensors 2002 Proceedings, Vol. 2, p. 1510-1515, 2002.

3. Hinkley, D., "Picosatellites at the Aerospace Corporation," Small Satellites: Past, Present, and Future, edited by H. Helvajian and S.W. Janson, The Aerospace Press/AIAA, El Segundo, CA, 2009, pp. 635-674.

4. Janson, S.W. and B.J. Barnhart, "The Next Little Thing: Femtosatellites," AIAA/Utah State Small Satellite Conference, Logan, UT, August 2013.

5. Janson, W. and Helvajian, H. "MEMS, Microengineering and Aerospace Systems," AIAA Paper 99-3802, 1999.

6. Magan, V., "British Nanosatellite Operated by a Smartphone Becomes World's First 'Phonesat'," Satellite Today, Vol. 12, No. 46, Mar 5, 2013.

7. Barnhart, D.J., Vladimirova, T., Baker, A.M., and M.N. Sweeting, "A Low-cost Femtosatellite to Enable Distributed Space Missions," Acta Astronautica, Vol. 64, No. 11-12, June-July 2009, pp. 1123-1143.

8. Barnhart, D.J., Vladimirova, T., and M.N. Sweeting, "Very-small-satellite Design for Distributed Space Missions," Journal of Spacecraft and Rockets, Vol. 44, No. 6, November 2007, pp. 1294-1306.

9. Balthazor, R.L., McHarg, M.G., Godbold, C.S., Barnhart, D.J., and T. Vladimirova,, "Distributed Space-based Ionospheric Multiple Plasma Sensor Networks,” 2009 IEEE Aerospace Conference, Big Sky, MT, March 2009.

10. Sundaramoorthy, P.P., Gill, E., and C.J.M. Verhoeven, "Systematic Identification of Applications for a Cluster of FemtoSatellites," 61st International Astronautical Congress, Prague, Czech Republic, September 2010.

11. Bouwmeester, J. and J. Guo, "Survey of Worldwide Pico- and Nanosatellite Missions, Distributions and Subsystem Technology," Acta Astronautica, Vol. 67, No. 7-8, October 2010, pp. 854-862.

12. Barnhart, D. J., et al., "Enabling Space Sensor Networks with PCBSat," $21^{\text {st }}$ Annual AIAA/USU Conference on Small Satellites, Logan, UT, August 2007.

13. Tristancho, J. and J. Gutierrez-Cabello, "A Probe of Concept of Femto-Satellite Based on Commercial Of-the-shelf," 2011 IEEE/AIAA 30 ${ }^{\text {th }}$ Digital Avionics Systems Conference, Seattle, October 2011.

14. Bell, I.C., et al., "Electrodynamic Tethers for ChipSat and Nanospacecrafts," Spacecraft Charging and Technology Conference, Kitakyushu, Japan, May 2012. 
15. Bell, I.C., Gilchrist, B.E., Bilén, S.G., and J.K. McTernan, "The Potential of Miniature Electrodynamic Tethers to Enhance Capabilities of Femtosatellites," 32nd International Electric Propulsion Conference, Wiesbaden, Germany, September 2011.

16. Bell, I.C., Gilchrist, B.E., Bilén, S.G., and J.K. McTernan, "Investigating the Use of Miniaturized Electrodynamic Tethers to Enhance the Capabilities of Femtosatellites and other Ultra-small Satellites," AIAA/Utah State Small Satellite Conference, Logan, UT, August 2012.

17. Bell, I.C., et al., "Investigating the Feasibility and Mission Enabling Potential of Miniaturized Electrodynamic Tethers for Femtosatellites and Other Ultra-small Satellites," AIAA/Utah State Small Satellite Conference, Logan, UT, August 2013.

18. Anguero, V.M., and Adamo, R.C., "Space Applications of Spindt Cathode Field Emission Arrays," 6th Spacecraft Charging Technology Conference, AFRL Science Center, Hancsom AFB, MA, 2000, pp. 347-352.

19. Ohkawa, Y., Kitamura, S., Kawamoto, S., Matsumoto, K., Kibe, S., Matsumoto, T., Murata, F., Matsui, M., and Yamagiwa, Y., "A Carbon Nanotube Field Emission Cathode for Electrodynamic Tether Systems," 32nd International Electric Propulsion Conference, Wiesbaden, Germany, 2011, pp. 1-10.

20. Crossley, B.L., "Carbon Nanotube Field Emission Arrays," Ph.D. Dissertation, Graduate School of Engineering and Management, Air Force Institute of Technology, Wright-Patterson Air Force Base, OH, 2011.

21. Barjatya, A., Swenson, C.M., Thompson, D.C., and K.H. Wright, "Data Analysis of the Floating Potential Measurement Unit Aboard the International Space Station,” Review of Scientific Instruments, Vol. 80, No. 4, April 2009.

22. McTernan, J.K., Bilen, S.G., Bell, I.C., and B.E. Gilchrist, "Current Collection to the Plasma Interaction with Femtosatellite- and Cubesat-Scale Electrodynamic Tether Subsystems," AIAA Space 2012 Conference, Pasadena, CA, Sept. 2012.

23. Wertz, J.R., Everett, F.D., and J.J. Puschell (eds.), Space Mission Analysis and Design, $3^{\text {rd }}$ ed., Microcosm, Inc., Hawthorne, CA, 2011.

24. Klofas, B., Anderson, J., and K. Leveque, "A Survey of CubeSat Communication Systems: 2009-2012," 10th Annual CubeSat Developers' Workshop, San Luis Obispo, CA, April 2013. 